\title{
State of the Art, Channel Assignment Multi-Radio Multi-Channel in Wireless Mesh Network
}

\author{
Ashraf Alzubir \\ Faculty of Computer \\ Science and Information \\ Systems \\ Universiti Teknologi \\ Malaysia
}

\author{
Kamalrulnizam Abu \\ Bakar \\ Faculty of Computer \\ Science and Information \\ Systems \\ Universiti Teknologi \\ Malaysia
}

\author{
Adil Yousif \\ Faculty of Computer \\ Science and Information \\ Systems \\ Universiti Teknologi \\ Malaysia
}

\author{
Albarraa Abuobieda \\ Faculty of Computer \\ Science and Information \\ Systems \\ Universiti Teknologi \\ Malaysia
}

\begin{abstract}
The emergence of new-generation networking technologies and the wide-spread use of computers and wireless devices are providing opportunities for a new generation of powerful network systems. Recently, many researchers proposed several channel assignment (CA) algorithms in multi-radio multi-channel assignment (MR-MCA) wireless mesh networks (WMNs) to utilize available channels set in the network topology architecture. One of the key challenges faces MR-MCA is how to reduce the interferences to ensure the maximum throughput by improving the aggregate network capacity. There are several studies that have classified these CA algorithms into several approaches. The aim of this paper is to discuss the current channel assignment approaches in MR-MCA in wireless mesh networks and highlighting the state of the art for MR-MCA, which can help researchers and developers in the field of WMNs.
\end{abstract}

\section{Keywords}

Wireless Mesh Network, Channel Assignment, Interference, Multi Channel, Multi Radio, Throughput.

\section{INTRODUCTION}

The wireless mesh networks are type of mobile ad hoc networks. WMNs are a collection of both fixed and mobile devices that are connected together by wireless links (channels) to form a multi-hop ad hoc network. The advantage of WMNs is their ability to fix several defects that are found in the network which are represented in the flexibility and connection reliability $[1,2]$. In addition, WMNs improved the limitation of other networks such as wireless ad hoc networks, wireless local area networks (WLANs) and wireless personal area network (WPANs) by covering large areas due to providing low cost internet access through multi-hop communications[2].

Typically, WMNs architecture is consisted of three levels: the top: Gateways, intermediate: Mesh routers and bottom level: Clients [3], as shown in Fig.1. The paragraphs below define each component separately:

Gateway serves as a bridge between wireless mesh network components and other networks such as the internet. In case the level of gateway absent or not available the WMN is used to serve in connecting local network only.

Mesh routers used to rout data packets from/to the other WMN components. Most of these routers are placed on the streetlight and on roof of houses[2], which serve as access points to cover large area as possible.
The mesh clients comprise any wireless device or stationary uses WMNs services, and may form a client mesh network among both themselves and mesh routers which often are laptops, cell phones, etc[4].

In WMNs each mesh router is equipped with several wireless network-interface cards (NICs) or radio interfaces, and each radio can be assigned to one orthogonal channel. Two radios can communicate and transmission with each other if they are assigned the same channel. The IEEE $802.11 \mathrm{~b} / \mathrm{g}$ and IEEE 802.11a standards provide three and twelve non-overlapping channels respectively[4].

CA is one of the important issues in multi-radio multi-channel in WMNs to ensure efficient utilization of channels available among mesh routers (nodes). The goal of channel assignment is to mitigates the interferences between channels and to ensure the number of paths available among the nodes (routers) to send the packages $[3,4]$.

In this paper, we will discuss the approaches of Channel Assignment in MR-MCA in WMNs which entails assigning a channel to each radio in order to ensure the efficient utilization of the available channels. The rest of this paper is organized as follows. Section 2 discusses the channel assignment in wireless mesh network. The taxonomy of channel assignment schemes extracted from the literature is presented in Section 3. Section 4 provides a discussion of Channel Assignment approaches. Finally, section 5 concludes the paper.

\section{CHANNEL ASSIGNMENT IN WMNS}

In order to improve the capacity of wireless mesh networks, each node in a network is equipped with multiple radio interfaces to assign multiple channels help increasing the throughput of network and minimize the interference [5]. In MR-MCA there are some considerations, in the following we present objectives and design Issues of MR-MCA in WMNs.

\subsection{The objectives of MR-MCA in WMNs} The main objectives of MR-MCA in WMNs are:

\subsubsection{Interference Minimization}

Interference is one of the important challenges that faces channel assignment in WMNs. When two nearby wireless links assigning same channels (same frequency), they cannot transmit data at the same time [6]. The interference is the foremost factor that degrades the wireless network performance. The primary goal of $\mathrm{CA}$ is to minimize interference in WMNs through utilizing multiple radios and multiple channels. There are two widely adopted models: Protocol Model and Physical Model: 


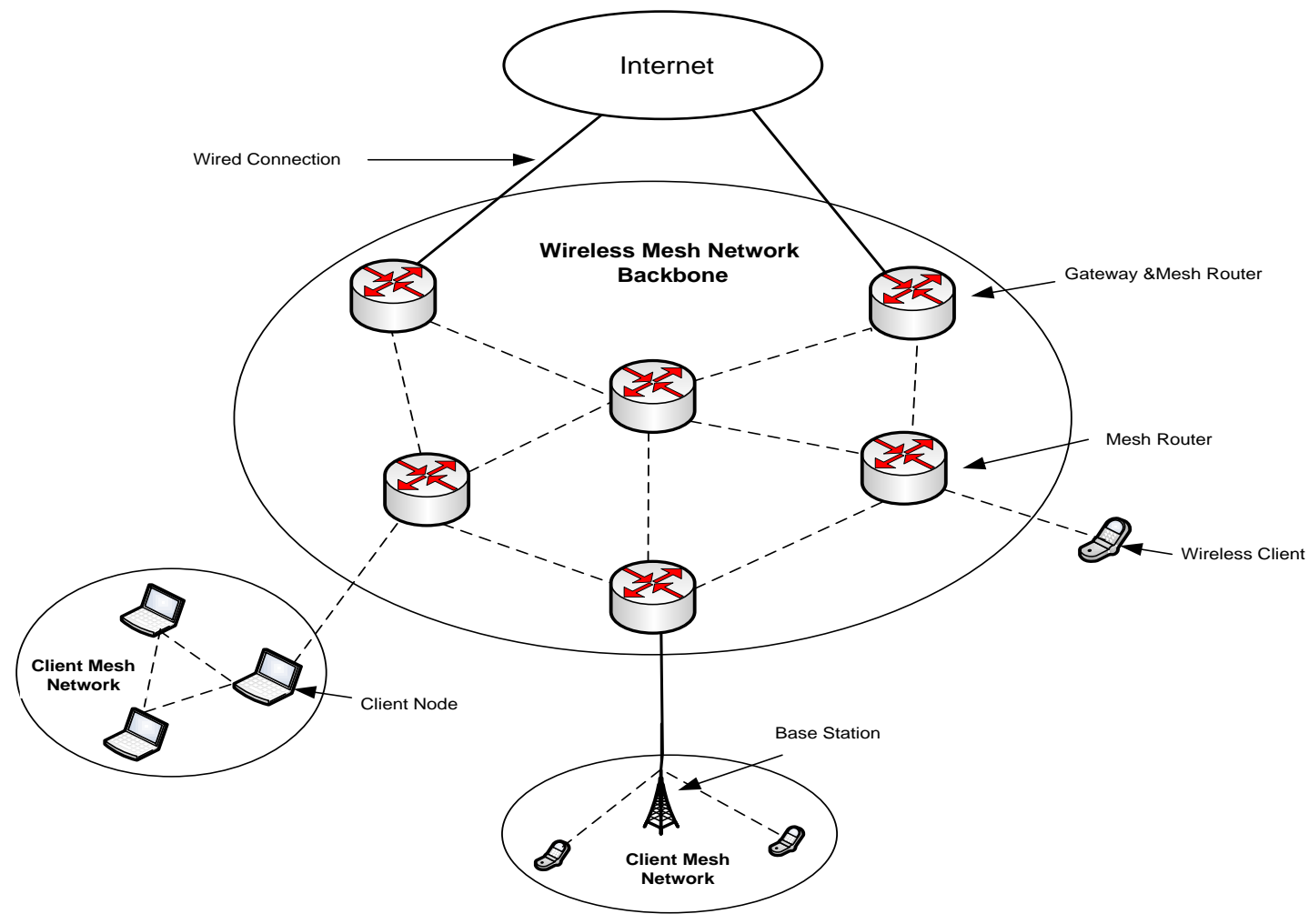

Fig 1: Wireless Mesh Network Architecture

Protocol Model can be described as follows: (1) each radio has a transmission range and an Interference range, with the former less than the latter; and (2) a transmission from radio $\mathrm{X}$ to radio $\mathrm{Y}$ is successful if $\mathrm{Y}$ is in the transmission range of $\mathrm{X}$ and not in the interference range of radios other than $\mathrm{X}$ that are currently transmitting.

Physical Model can be described as follows: (1) a transmission is successful if the Signal of Interference and Noise Ratio (SINR) of the transmitter at the receiver is larger than a threshold value; and (2) the interference and noise power at the receiver consists of noises generated by other ongoing transmissions and the ambient noise in the network.

\subsubsection{Throughput maximization}

Most researchers recently focus on using MR-MCA to improve the network throughput in WMNS by increasing the number of parallel transmission in the network[7].

\subsubsection{Load balancing}

The objective of load balance is to assign channels to nodes meanwhile take into account the traffic load balance among channels in the network and mitigate the interference[8].

\subsection{Design Issues in Multi-Channel Assignment}

In WMNs there are some key design issues in MR-MCA should be considered [3]:

\subsubsection{Maintaining connectivity}

Maintaining the network structure to avoid separation of the network into sub-networks is useful for network resources; thereby, enhancing the performance of the network through the exchange of information and ease of maintenance operations. Many advantages are originated from connectivity of WMNs to ensure reliable mesh connectivity, network selforganization and topology control algorithms[9].

\subsubsection{Routing}

Routing in some cases depends on CA and vice versa Recently, the researcher's trends to design joint routing and CA schemes that optimize the route by selecting the channels along the end to end path. To successfully installing Routing protocol is to avoid congestion and minimize the interference in the network[9].

\subsubsection{Fault tolerance}

The fault tolerance is an autonomous solution process issued by the router due to unexpected fails in software and/or hardware components such as, router to router disconnection due to external interference or temporary obstacles. So it is necessary for CA approach to support fault tolerance such that the network can operate in self-healing fashion[3].

\subsubsection{Fairness}

Fairness in CA is the ability of the CA algorithms in organizing the overall traffic load between the nodes of the network[10].

\section{APPROACHES OF MULTI- CHANNEL ASSIGNMENT FOR MULTI- RADIO}

Channel assignment approaches in WMNs are proposed to improve the throughput and mitigate the interference by efficient utilization of channels[11]. There are many categories in multi-channel assignment for multi-radio in WMNs; in this paper we will introduce the two most important categories (see Fig.2). First, channel assignment based on available knowledge on channels, in which its approaches are classified into Centralized and Distributed 


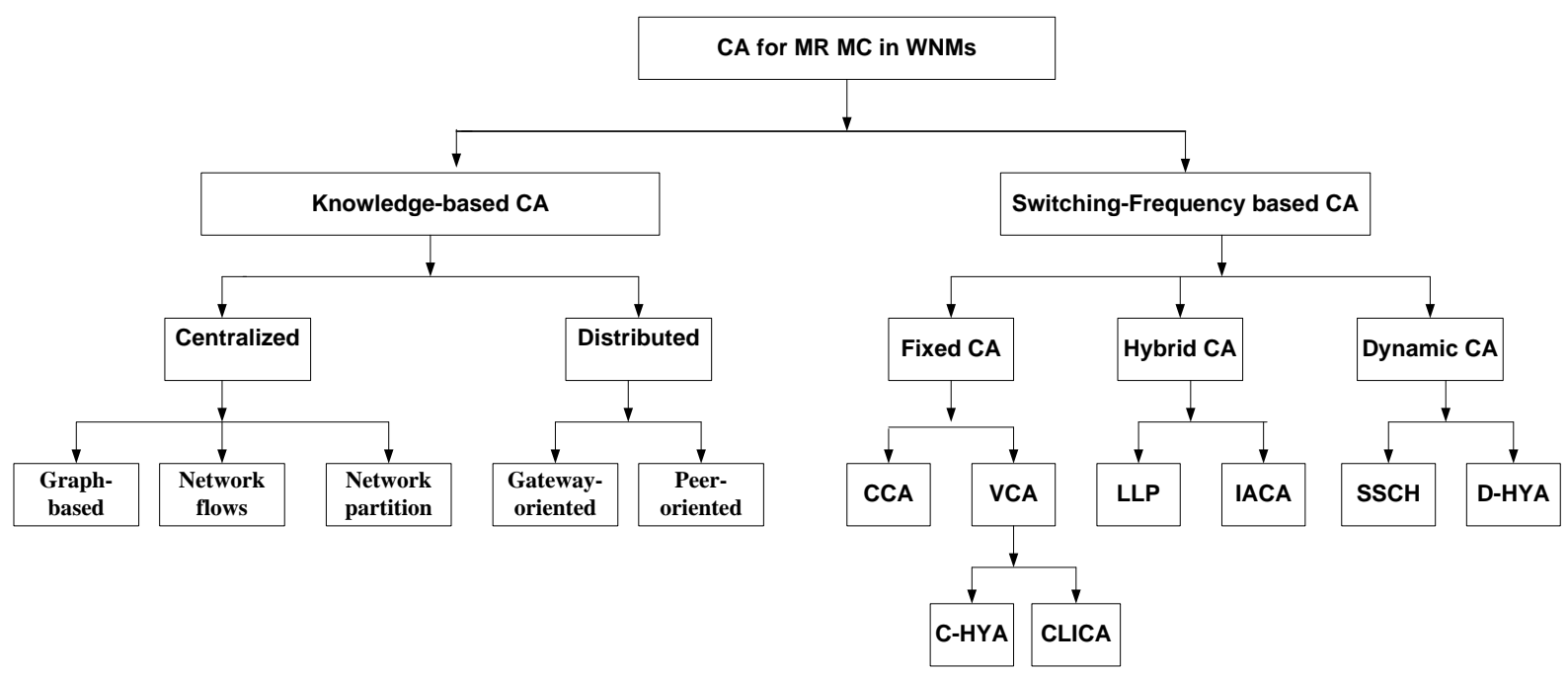

Fig 2: Classification of CA approaches for MR-MC WMNs

channel assignment[12]. Second, channel assignment based on frequency, in which its schemes are classified into Fixed/Static, Dynamic and Hybrid Channel Assignment[11].

\subsection{Knowledge-based Channel Assignment (KCA)}

In this section, we can divide the channel assignment approaches into two classes: Centralized and Distributed channel assignment. These approaches are relying on the methods for collecting information on the channels to be assigned[3].

\subsubsection{Centralized Channel Assignment}

Each node in WMN sends its current channel(s) information status(es) to a central unite. This information include: 1) interference level, 2) default channel available, and 3) assigned channel. A centralized unit algorithm for channel assignment uses these information to compose a global knowledge about the WMN topology, which can be used later to assign channels to radios. All nodes send their channel information (all the channels assigned to radios and channels still available) to the central entity which assigns channels for nodes based on this information. The main weakness of this category is that, the network fails completely, when the channel assignment server is failed. The centralized approaches are classified into three categories according to their problem formulations: the graph-based approaches, the network flows approaches, and the network partitioning approaches[3].

\subsubsection{Graph-based approaches}

The concepts of MR-MCA in WMNs are modeled by a graph with a vertex set and an edge set. A conflict graph is one of these approaches used in CA problem. In the conflict graph concept, we use the terms "vertex" and "edge" Instead of "node" and "link" as in the connectivity graph[3]. For instance, the link between nodes A and B in the connectivity graph in Fig.3 (a) is represented by a vertex $1 \mathrm{AB}$ in the conflict graph of Fig.3 (b). An edge is placed between two vertices in the conflict graph if the corresponding links in the connectivity graph interfere.

\subsubsection{Network Flows Approaches}

The network flow is formulated in CA problem by all the approaches in this category assume the traffic rates (either end-to-end or at each source) are known. Some researchers have used this approach, such as Murali and Thyaga in [13], Mansoor, Randeep and Li in [14] and Ashish, Kartik and Tzi-cker in [15]. One of the advantages of this method, which is characterized by the previous method (graph-based approaches), is includes the traffic load information, which is neglected by graph-based approaches. And the most limitations of their approaches assume constant traffic rates, which is not realistic in most practical networks where the traffic pattern is usually burst and characterized by random on/off sources.

\subsubsection{Network Partition Approaches}

Some researches such as [16] [17], tried to solve the problem of CA approach by divide the network into a set of separate sub networks so that all the radios and links in MR-MCA in WMN are divided into separate sub networks so that they use each sub networks as a single channel. To reduce the problem of interference, a set of different channels are assigned to these sub networks. The Advantage of network partitioning methodology is straightforward and simple, resulting in polynomial-time problems instead of NP-hard problems. In addition, the disadvantages of these approaches in this category are all links in a partition are fixed to a common channel, making the network is inflexible, which leads to decrease the network throughput and reduce the interference in the network.

\subsubsection{Distributed Channel Assignment}

The distributed approach is more feasible in realistic environments where the global information for centralized algorithm is not available. On the other hand, in distributed CA each node measures local channel information and exchanges it with other nodes to calculate the channel assignment[18]. Based on distributed CA in WMNs there are several classes as the following:

\subsubsection{Gateway-Oriented Approaches}

Gateway-oriented approaches are one of the methods which coordinate the network traffic across the central sinks through 


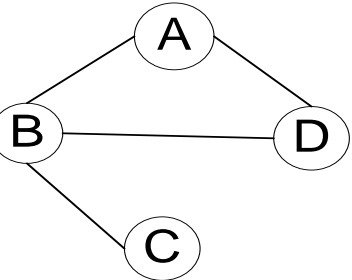

Fig 3(a): Connectivity graph

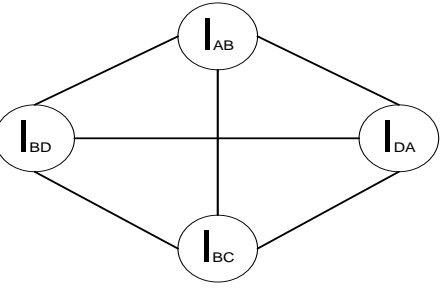

Fig 3(b): Conflict graph the appointment of certain number of gateways in the network. In [19-21] the researchers formulated the CA problem into concept of Gateway-oriented approaches. The advantage of these approaches is the opportunity to utilize the gateway nodes to simplify the CA approach. Gatewayoriented approaches limitation is the incapability of accommodating other kinds of traffic patterns.

\subsubsection{Peer-Oriented Approaches}

Peer-oriented approach is differ than previous methods as it accommodates different types of network traffic and do not make any restrictions on them, which makes it flexible to be used in the networks. One of the drawbacks of these approaches is that, the CA is difficult to fault tolerance. In [1, $22,23]$ the researchers have benefited from the properties of the Peer-oriented approach in the process of CA. The benefit is the capability of adapting to various kinds of traffic patterns, making it applicable for most of the current WMNs, where the peer-to-peer traffic is also a major part of the total network traffic.

\subsection{Switching-Frequency based Channel Assignment (SFCA)}

In this section, we describe different schemes that can be used to assign channels in a wireless mesh network. The classification is based on channel switching frequency. We can divide these schemes into three classes' strategies: Fixed/Static, Dynamic and Hybrid channel assignment. Next subsections contain the details about these categories.

\subsubsection{Fixed/Static Channel Assignment}

Fixed or Static channel assignment strategies are assigning each radio interface in the node to an available channel either permanently, or for long time durations. There are two different schemes in this strategy as follows[24]:

\subsubsection{Common Channel Assignment (CCA)}

In this case the radio interfaces of each node are all assigned the same set of channels. This type of scheme is the simplest types of CA in WMNs. The main benefit is that the connectivity of the network is same for each connection. The limitation of this scheme is it does not utilize the network resources effectively and furthermore it does not care about the factors that affect network performance[4]

\subsubsection{Varying Channel Assignment (VCA)}

Methods of CA in VCA may lead to the partitions in the network and topology changes that may increase the length of routes between the mesh nodes in the process of assigned different channels to a set of the radio interfaces in different nodes[4]. This paper in the flowing describes the VCA approach through two existing algorithms.

\subsection{Centralized Channel Assignment (C- \\ HYA)}

In[15], based on Hyacinth, centralized channel assignment algorithm for WMNs the traffic is directed to/from the Internet. Assuming that the offered traffic load is known, this algorithm assigns channels thus ensuring network connectivity and satisfying the bandwidth limitations of each link. Its first estimates the total expected load on each virtual link by summing the load due to each offered traffic flow. Then, the channel assignment algorithm visits each virtual link in decreasing order of expected traffic load and greedily assigns it a channel. The algorithm starts with an initial estimation of the expected traffic load and iterates over channel assignment and routing until the bandwidth allocated to each virtual link matches its expected load. Although this scheme presents a method for channel allocation that incorporates connectivity and traffic patterns, the assignment of channels on links may cause a ripple effect whereby, already assigned links have to be revisited, thus increasing the time complexity of the scheme.

\subsection{Connected Low Interference Channel}

\section{Assignment (CLICA)}

CLICA is a traffic independent channel assignment scheme which computes the priority for each mesh node and assigns channels based on the connectivity graph and the conflict graph[25]. However, the algorithm can override the priority of a node to account for the lack of flexibility in terms of channel assignment and to ensure network connectivity. Although this scheme avoids link revisits, it does not incorporate the role of traffic patterns in channel assignment for WMNs. The benefits of the CLICA are enabling an efficient and flexible topology formation; ease of coordination, and to exploit the static nature of mesh routers to update the channel assignment on large timescales.

\subsubsection{Dynamic Channel Assignment}

In dynamic CA schemes, any radio interface can be assigned to any channel. In addition, radios can frequently switch from one channel to another. Therefore, when nodes need to communicate with each other in such a scheme, a coordination mechanism is required to ensure that they are on a common channel. 
Table 1. Comparative study of channel assignment approaches and schemes

\begin{tabular}{|c|c|c|c|c|}
\hline $\begin{array}{c}\text { Author \& } \\
\text { date }\end{array}$ & Approach & technique used & The Aim & Metrics \\
\hline $\begin{array}{l}\text { A.Subramani } \\
\text { an, H. Gupta, } \\
\text { S. Das [1] }\end{array}$ & $\begin{array}{l}\text { Centralized and } \\
\text { Distributed CA }\end{array}$ & $\begin{array}{c}\text { Centralized Tabu-based Algorithm } \\
\text { (CTA) }\end{array}$ & $\begin{array}{l}\text { To minimizing network } \\
\text { interference }\end{array}$ & $\begin{array}{c}\text { Interfaces } \\
\text { \&Throughput }\end{array}$ \\
\hline $\begin{array}{l}\text { M. Kodialam, } \\
\text { T. } \\
\text { Nandagopal } \\
{[13]} \\
\end{array}$ & Centralized CA & $\begin{array}{c}\text { Balanced Static Channel Assignment } \\
\text { \& Packing Dynamic Channel } \\
\text { Assignment (BSCA\&PDCA) }\end{array}$ & $\begin{array}{c}\text { Enhance traffic loads and link } \\
\text { capacity }\end{array}$ & $\begin{array}{l}\text { Number of } \\
\text { channel \& } \\
\text { capacity }\end{array}$ \\
\hline $\begin{array}{l}\text { S. Avallone, } \\
\text { I.F. Akyildiz } \\
\text { [16] }\end{array}$ & Centralized CA & $\begin{array}{c}\text { Max flow-based Centralized Channel } \\
\text { Assignment (MCCA) }\end{array}$ & To maximize the network capacity & $\begin{array}{c}\text { Available } \\
\text { channel \& } \\
\text { Throughput } \\
\end{array}$ \\
\hline $\begin{array}{l}\text { Avallone and } \\
\text { Akyildiz in } \\
{[16]}\end{array}$ & Centralized CA & $\begin{array}{l}\text { Integer linear programming (ILP) } \\
\text { model }\end{array}$ & Maximizing the network capacity & Throughput \\
\hline $\begin{array}{l}\text { Rahman, } \\
\text { Agarwal et al } \\
\text { in [12] }\end{array}$ & Distributed CA & $\begin{array}{l}\text { Design algorithm to select channel } \\
\text { through capacity and interference }\end{array}$ & $\begin{array}{l}\text { Choosing the best channel among } \\
\text { all the available channels. }\end{array}$ & $\begin{array}{l}\text { Throughput \& } \\
\text { Delay }\end{array}$ \\
\hline $\begin{array}{l}\text { Sridhar, Guo } \\
\text { et al. in [26] }\end{array}$ & Distributed CA & $\begin{array}{l}\text { Graph-theoretic and Integer linear } \\
\text { programming (ILP) model }\end{array}$ & $\begin{array}{l}\text { Assigning channels to radio } \\
\text { interfaces in multi-radio, multi- } \\
\text { channel }\end{array}$ & $\begin{array}{c}\text { Total } \\
\text { Interference }\end{array}$ \\
\hline $\begin{array}{l}\text { amamurthi, } \\
\text { Reaz et al in } \\
{[27]}\end{array}$ & Distributed CA & Linear Programming (LP) & $\begin{array}{c}\text { Enhance the effectiveness of } \\
\text { WMNs }\end{array}$ & $\begin{array}{c}\text { Delay \& } \\
\text { Throughput }\end{array}$ \\
\hline $\begin{array}{l}\text { Luong, Lee et } \\
\text { al in [28] }\end{array}$ & $\begin{array}{l}\text { Distributed channel } \\
\text { assignment and } \\
\text { Routing protocol }\end{array}$ & $\begin{array}{l}\text { Ad-hoc on-demand distance vector } \\
\text { modification (AODV-M) }\end{array}$ & $\begin{array}{l}\text { Forwarding packets based on data } \\
\text { traffic }\end{array}$ & $\begin{array}{c}\text { Delay \& } \\
\text { Throughput }\end{array}$ \\
\hline $\begin{array}{l}\text { Mirzaie, } \\
\text { Sarram et } \\
\text { al.in [29] }\end{array}$ & Static MR-MCA & Genetic algorithm and Tabu search & $\begin{array}{l}\text { Maximizing the available network } \\
\text { throughput by minimizing the } \\
\text { network interference. }\end{array}$ & $\begin{array}{l}\text { Interference and } \\
\text { throughput }\end{array}$ \\
\hline $\begin{array}{l}\text { Bin, Zhifeng } \\
\text { et al.in [30] }\end{array}$ & Static CA & Integer Quadratic Programming (IQP) & $\begin{array}{c}\text { To minimize the average network } \\
\text { interference }\end{array}$ & $\begin{array}{c}\text { Interfaces \& } \\
\text { radios } \\
\end{array}$ \\
\hline $\begin{array}{l}\text { Makram, } \\
\text { S.A., et al } \\
\text { [31] }\end{array}$ & Dynamic CA & Cluster Channel Assignment (CCA) & $\begin{array}{c}\text { Maximize the aggregate } \\
\text { throughput, Capacity and } \\
\text { Minimizing the interference } \\
\end{array}$ & $\begin{array}{l}\text { Throughput \& } \\
\text { Time }\end{array}$ \\
\hline $\begin{array}{l}\text { Ding, } \\
\text { Pongaliur et } \\
\text { al. in [32] }\end{array}$ & Hybrid CA & $\begin{array}{c}\text { Adaptive Dynamic Channel } \\
\text { Allocation protocol (ADCA) \& } \\
\text { Interference and Congestion Aware } \\
\text { Routing protocol (ICAR) } \\
\end{array}$ & $\begin{array}{l}\text { First to optimization for both } \\
\text { throughput and delay in the channel } \\
\text { assignment. Second to balances the } \\
\text { channel usage in the network }\end{array}$ & $\begin{array}{l}\text { Delay \& } \\
\text { Throughput }\end{array}$ \\
\hline $\begin{array}{l}\text { Lave, } x \text { et al } \\
\quad \text { in [33] }\end{array}$ & Hybrid CA & Novel Routing Mechanism & $\begin{array}{l}\text { Reducing the switching overhead in } \\
\text { hybrid multichannel multi-radio } \\
\text { wireless mesh networks }\end{array}$ & Delay \& Loads \\
\hline
\end{tabular}

The key challenge with the dynamic switching approach is how to coordinate decisions when the radios can frequently switch from one channel to another and how to choose the appropriate channel.

\subsubsection{Slotted Seeded Channel Hopping (SSCH)}

$\mathrm{SSCH}$ uses pseudo-random sequences [34] where each node should switch channels synchronously in a pseudo-random sequence so that all neighbors meet periodically in the same channel. In this approach the interfaces must be capable of fast synchronous channel switching. Specifically, time is divided into slots and the channels are switched at the beginning of each slot.

\subsubsection{A Distributed Channel Assignment}

\section{Scheme (D-HYA)}

A set of dynamic and distributed channel assignment algorithms was proposed in $[35,36]$ based on Hyacinth. These algorithms can react to traffic load changes in order to improve the aggregate throughput and achieve load balancing.
Based on the Hyacinth architecture, the previous algorithm builds on a spanning tree network topology, the gateway node is the root of a spanning tree, and every mesh node belongs to that tree. The channel assignment problem consists of the following two steps.

a) Neighbor-to-interface binding which determines the interface that a node should use to communicate with each of its neighbors with whom it intends to establish a link.

b) Interface-to-channel binding determines which radio channel a network interface should use.

Based on per-channel total load information, a WMN node determines the set of channels that are least used in its vicinity. As nodes higher up in the spanning trees need more relay bandwidth, they are given a higher priority in channel assignment.

\subsubsection{Hybrid Channel Assignment}

Hybrid channel assignment schemes combine both static and dynamic assignment properties by applying a fixed 
assignment for some radios and a dynamic assignment for other radios. The fixed radios can be assigned to dedicated channels while the other radios can be switched dynamically among channels. In the following, we describe two hybrid schemes for CA[24].

\subsubsection{Link Layer Protocols for Radio Assignment (LLP)}

In [37, 38], an innovative link layer radio assignment algorithm was proposed that categorizes available radios into fixed (F) and switchable (S) radios. Fixed radios are assigned, for long time intervals, specific fixed channels, which can vary for different nodes. On the other hand, switchable radios can be switched over short time scales among the non-fixed channels based on the amount of data traffic. By distributing fixed radios of different nodes on different channels, all channels can be used, while the switchable radio can be used to maintain the connectivity.

\subsubsection{Interference-Aware Channel Assignment (IACA)}

To improve the capacity of the network and to reduce the interference, the researchers in [39] have proposed IACA algorithm. This algorithm is based on an extension to the conflict graph concept, which called the multi-radio conflict graph (MCG), where the vertices in the MCG represent edges between mesh radios instead of edges between mesh routers. In this algorithm proposed assigns one radio on each node to operate on a default common channel throughout the network that for ensures a common network connectivity graph, provides alternate fallback routes, and avoids flow disruption by traffic redirection over a default channel. The channel assignment works on the Breadth First Search Channel Assignment (BFS-CA) algorithm based on rank strategy, where each router has two separate channel rankings (increasing number of interfering radios and increasing channel utilization)

\section{DISCUSSION}

$\mathrm{CA}$ is one of the important issues in WMN, and it needs to be improved and developed continuously to meet the challenges and requirements of the recent technologies. This makes CA an interested and hot area, which motivated the researchers to present different classifications based on several criteria, as described in the previews sections to find solution to the problems facing the performance of networks.

Based on the various taxonomies and different approaches in $\mathrm{CA}$, we have come up with the following table. According to table 1 most of CA approaches have the following concerns. First, they try to reduce the interference problem, which is one of the fundamental problems that hinder the performance of the network to send packets from the source to the destination. Second, the majority of the researches strive to increase the network throughput by using parallel transmission of packets and avoiding congestion, which helps in improving the performance of the WMN.

\section{CONCLUSION}

The WMNs have evolved from single-radio single-channel architecture to MR-MC architecture. MR-MC architecture launches a new research area in channel assignment, which seeks to find the optimal mapping between the channels and radios at each node to improve the network performance. This paper reviewed the objectives, classifications of existing channel assignment schemes and approaches as well as discussed the current channel assignment approaches in MR-
MCA in wireless mesh networks. Furthermore, the paper highlighted the state of the art for MR-MCA, which can help researchers and developer in the field of WMNs.

\section{ACKNOWLEDGEMENTS}

The authors would like to thank Universiti Teknologi Malaysia for the unlimited support. We also appreciate the significant role of Sudanese Students Research Group (SSRGUTM) to adopt this work from ideas to reality.

\section{REFERENCES}

[1] Subramanian, A.P., H. Gupta, and S.R. Das. Minimum Interference Channel Assignment in Multi-Radio Wireless Mesh Networks. in Sensor, Mesh and Ad Hoc Communications and Networks, 2007. SECON '07. 4th Annual IEEE Communications Society Conference on. 2007.

[2] Akyildiz, I.F., X. Wang, and W. Wang, Wireless mesh networks: a survey. Computer networks, 2005. 47(4): p. 445-487.

[3] Si, W., S. Selvakennedy, and A.Y. Zomaya, An overview of channel assignment methods for multi-radio multichannel wireless mesh networks. Journal of Parallel and Distributed Computing, 2010. 70(5): p. 505-524.

[4] Conti, M., et al., Channel Assignment Strategies for Wireless Mesh Networks, in Wireless Mesh Networks, E. Hossain and K. Leung, Editors. 2007, Springer US. p. 113-142.

[5] Tran Minh, T., G. Hong Yong, and S. Park Jeong. A load aware hybrid channel assignment for Multi-radio Wireless Mesh Network. in Optical Internet (COIN), 2010 9th International Conference on. 2010.

[6] Subramanian, A.P., et al., Minimum interference channel assignment in multiradio wireless mesh networks. IEEE Transactions on Mobile Computing, 2008: p. 1459-1473.

[7] LEI, H., et al., Survey of multi-channel MAC protocols for IEEE 802.11-based wireless Mesh networks. The Journal of China Universities of Posts and Telecommunications, 2011. 18(2): p. 33-44.

[8] Bokhari, F. and G. Zaruba. AMIRA: Interference-Aware Routing Using Ant Colony Optimization in Wireless Mesh Networks. in Wireless Communications and Networking Conference, 2009. WCNC 2009. IEEE. 2009.

[9] Xiaoguang, L. and X. Changqiao. Joint Channel Assignment and Routing in Real Time Wireless Mesh Network. in Wireless Communications and Networking Conference, 2009. WCNC 2009. IEEE. 2009.

[10] Mohsenian-Rad, A.H. and V.W.S. Wong, Joint logical topology design, interface assignment, channel allocation, and routing for multi-channel wireless mesh networks. Wireless Communications, IEEE Transactions on, 2007. 6(12): p. 4432-4440.

[11] Skalli, H., et al., Channel assignment strategies for multiradio wireless mesh networks: issues and solutions. Communications Magazine, IEEE, 2007. 45(11): p. 8695 .

[12] Rahman, M., A. Agarwal, and A. Alsarahn. Capacity based channel assignment in multi-interface wireless mesh networks. 2008: IEEE. 
[13] Kodialam, M. and T. Nandagopal, Characterizing the capacity region in multi-radio multi-channel wireless mesh networks, in Proceedings of the 11th annual international conference on Mobile computing and networking. 2005, ACM: Cologne, Germany. p. 73-87.

[14] Alicherry, M., R. Bhatia, and L. Li, Joint channel assignment and routing for throughput optimization in multi-radio wireless mesh networks, in Proceedings of the 11th annual international conference on Mobile computing and networking. 2005, ACM: Cologne, Germany. p. 58-72.

[15] Raniwala, A., K. Gopalan, and T. Chiueh, Centralized channel assignment and routing algorithms for multichannel wireless mesh networks. ACM SIGMOBILE Mobile Computing and Communications Review, 2004. $8(2)$ : p. $50-65$.

[16] Avallone, S. and I.F. Akyildiz, A channel assignment algorithm for multi-radio wireless mesh networks. Computer Communications, 2008. 31(7): p. 1343-1353.

[17] Brzezinski, A., G. Zussman, and E. Modiano, Enabling distributed throughput maximization in wireless mesh networks: a partitioning approach, in Proceedings of the 12th annual international conference on Mobile computing and networking. 2006, ACM: Los Angeles, CA, USA. p. 26-37.

[18] Hong, X., et al., Exploring multiple radios and multiple channels in wireless mesh networks [Accepted from Open Call]. Wireless Communications, IEEE, 2010. 17(3): p. 76-85.

[19] Das, S.M., et al., DMesh: Incorporating Practical Directional Antennas in Multichannel Wireless Mesh Networks. Selected Areas in Communications, IEEE Journal on, 2006. 24(11): p. 2028-2039.

[20] 20. Naveed, A., S.S. Kanhere, and S.K. Jha. Topology Control and Channel Assignment in Multi-Radio MultiChannel Wireless Mesh Networks. in Mobile Adhoc and Sensor Systems, 2007. MASS 2007. IEEE Internatonal Conference on. 2007.

[21] Raniwala, A. and T. Chiueh. Architecture and algorithms for an IEEE 802.11-based multi-channel wireless mesh network. 2005: IEEE.

[22] 22. Bong-Jun, K., et al. Distributed Channel Assignment in Multi-Radio 802.11 Mesh Networks. in Wireless Communications and Networking Conference, 2007.WCNC 2007. IEEE. 2007.

[23] Xing, K., et al., Superimposed code based channel assignment in multi-radio multi-channel wireless mesh networks, in Proceedings of the 13th annual ACM international conference on Mobile computing and networking. 2007, ACM: Montrl\&\\#233;al, Qul\&\\#233;bec, Canada. p. 15-26.

[24] 24. Kyasanur, P. and N.H. Vaidya. Routing and interface assignment in multi-channel multi-interface wireless networks. in Wireless Communications and Networking Conference, 2005 IEEE. 2005.

[25] Marina, M.K., S.R. Das, and A.P. Subramanian, A topology control approach for utilizing multiple channels in multi-radio wireless mesh networks. Computer networks, 2010. 54(2): p. 241-256.
[26] Sridhar, S., J. Guo, and S. Jha. Channel assignment in multi-radio wireless mesh networks: a graph-theoretic approach. 2009: IEEE.

[27] Ramamurthi, V., et al., Channel, capacity, and flow assignment in wireless mesh networks. Computer networks, 2011. 55(9): p. 2241-2258

[28] 28. Luong, T.T., et al. Efficient channel assignment and routing protocol for multiple channels multiple interfaces wireless mesh network: IEEE.

[29] Mirzaie, S., M.A. Sarram, and V. Derhami. Throughput enhancement via channel assignment algorithms within Wireless Mesh Networks. in Wireless Communication and Sensor Networks (WCSN), 2010 Sixth International Conference on. 2010

[30] Bin, Z., et al. Optimization model for static channel assignment in multi-radio multi-channel wireless mesh networks. in Wireless Communications \& Signal Processing, 2009. WCSP 2009. International Conference on. 2009.

[31] Makram, S.A., et al. Dynamic Channel Assignment for Wireless Mesh Networks Using Clustering. in Networking, 2008. ICN 2008. Seventh International Conference on. 2008.

[32] Yong, D., K. Pongaliur, and X. Li. Hybrid multi-channel multi-radio wireless mesh networks. in Quality of Service, 2009. IWQoS. 17th International Workshop on. 2009.

[33] Lave, et al. Multi-channel anypath routing in wireless mesh networks. in GLOBECOM Workshops (GC Wkshps), 2010 IEEE. 2010.

[34] Bahl, P., R. Chandra, and J. Dunagan, SSCH: slotted seeded channel hopping for capacity improvement in IEEE 802.11 ad-hoc wireless networks, in Proceedings of the 10th annual international conference on Mobile computing and networking. 2004, ACM: Philadelphia, PA, USA. p. 216-230.

[35] Raniwala, A. and C. Tzi-cker. Evaluation of a wireless enterprise backbone network architecture. in High Performance Interconnects, 2004. Proceedings. 12th Annual IEEE Symposium on. 2004

[36] Raniwala, A. and C. Tzi-cker. Architecture and algorithms for an IEEE 802.11-based multi-channel wireless mesh network. in INFOCOM 2005. 24th Annual Joint Conference of the IEEE Computer and Communications Societies. Proceedings IEEE. 2005.

[37] Kyasanur, P. and N.H. Vaidya, Routing and link-layer protocols for multi-channel multi-interface ad hoc wireless networks. ACM SIGMOBILE Mobile Computing and Communications Review, 2006. 10(1): p. 31-43.

[38] Kyasanur, P. and N.H. Vaidya. Routing and interface assignment in multi-channel multi-interface wireless networks. 2005: IEEE.

[39] Ramachandran, K.N., et al. Interference-Aware Channel Assignment in Multi-Radio Wireless Mesh Networks. in INFOCOM 2006. 25th IEEE International Conference on Computer Communications. Proceedings. 2006. 